\title{
Chaperonin-containing T-complex protein 1 subunit 8 promotes cell migration and invasion in human esophageal squamous cell carcinoma by regulating $\alpha$-actin and $\beta$-tubulin expression
}

\author{
XIAOJING YANG ${ }^{1 *}$, HANRU REN $^{2 *}$, YUHUI SHAO ${ }^{1}$, YI SUN ${ }^{1}$, LIHUA ZHANG $^{1}$, HONGLING LI $^{1}$, \\ XIULONG ZHANG ${ }^{1}$, XINMIAO YANG ${ }^{1}$, WEIWEI YU ${ }^{1}$ and JIE FU ${ }^{1}$ \\ ${ }^{1}$ Department of Radiation Oncology, Shanghai Jiao Tong University Affiliated with Sixth People's Hospital, \\ Shanghai 200233; ${ }^{2}$ Department of Orthopedics, Shanghai Pudong Hospital, Fudan University, \\ Pudong Medical Center, Shanghai 201300, P.R China
}

Received November 26, 2017; Accepted March 15, 2018

DOI: $10.3892 /$ ijo.2018.4335

\begin{abstract}
The chaperonin-containing T-complex protein 1 (CCT) has eight subunits, CCT 1-8, which are dysregulated in several types of cancer. To determine how subunit 8 (CCT8) influences the development of esophageal squamous cell carcinoma (ESCC), immunohistochemistry and western blot analysis were performed on 128 ESCC samples in the present study to measure the expression of CCT8. The prognostic value of CCT8 was analyzed using univariate and multivariate survival analyses. CCT8 knockdown in ESCC cells was performed and subsequently, the migration and invasion of ESCC cells was assessed. The results of immunohistochemistry and western blot analysis of ESCC tissue indicated that the expression of CCT8 in tumor tissues from patients with lymph node metastasis (LNM) was high whereas its expression in tissues from those without LNM was low. In addition, the overall survival rate of patients with high CCT8 expression was poor. It was demonstrated that CCT8 influenced the migration and invasion of ESCC cells by regulating $\alpha$-actin and $\beta$-tubulin. Following CCT8 knockdown, cells were treated with cisplatin; it was demonstrated that $\alpha$-actin and $\beta$-tubulin were downregulated and that cell apoptosis was enhanced. These data confirm that $\alpha$-actin and $\beta$-tubulin are regulated by CCT8, and that increased CCT8 expression is associated with poor patient prognosis and cisplatin resistance in ESCC.
\end{abstract}

Correspondence to: Dr Jie Fu, Department of Radiation Oncology, Shanghai Jiao Tong University Affiliated Sixth People's Hospital, 600 Yishan Road, Shanghai 200233, P.R. China

E-mail: fujie74@sjtu.edu.cn

${ }^{*}$ Contributed equally

Key words: chaperonin-containing T-complex protein 1 subunit 8, esophageal squamous cell carcinoma, migration, invasion, prognosis

\section{Introduction}

Esophageal squamous cell carcinoma (ESCC) is a type of malignant gastroenterological tumor with a high incidence in China (1). ESCC tumors readily undergo metastasis and this is the primary explanation for the poor prognosis of patients with ESCC (2). Cancer metastasis is associated with compound mechanisms, sunch as epithelial-mesenchymal transition (EMT). The expression of E-cadherin, an epithelial marker, is decreased in EMT (3). It is important to determine the process by which ESCC tumors undergo metastasis, in order to improve the diagnosis of patients with ESCC and develop effective therapeutic strategies to treat this disease (4).

It has been demonstrated that oncogenes are associated with tumorigenesis, such as epidermal growth factor receptor (EGFR), cyclin D1 and p53 (5). The chaperonin containing T-complex protein 1 (CCT) contains eight different subunits, CCT 1-8 (6). CCT is required to stimulate the folding of actin, tubulin and other cytosolic proteins $(7,8)$ and is involved in mediating various biological processes, including cytoskeletal organization $(9-11)$, cell cycle progression $(9,11)$ and apoptosis (12). Yokota et al (13) initially identified CCT as an oncogene; they reported that CCT was highly expressed in liver and colon cancer. It has since been demonstrated that CCT expression is correlated with the growth of mammary carcinoma cells $(14,15)$. Several studies have indicated that CCT, particularly subunit 8 (CCT8) serves an important role during the progression of tumors, including B-cell non-Hodgkin's lymphoma (16), glioma (17) and hepatocellular carcinoma (18). However, to the best of our knowledge, the expression of CCT8 in ESCC and the role it serves remain unclear.

The goal of the present study was to determine the association between CCT8 and ESCC and assess the feasibility of using CCT8 as a novel prognostic indicator in ESCC. The functional role of CCT8 in the migration and invasion of ESCC cells was examined following transfection with small interfering (si)RNA to induce CCT8 knockdown. The results 
demonstrate that CCT8 enhances ESCC cell migration and invasion by regulating the expression of $\alpha$-actin and $\beta$-tubulin.

\section{Materials and methods}

Tissue samples and cell culture. Tissue specimens were acquired from 128 patients (mean age, 66 years; $68.0 \%$ male) with ESCC who underwent cancer resection at Shanghai Jiao Tong University Affiliated with Sixth People's Hospital between July, 2005 and June, 2011. Patients had received no treatment for ESCC prior to surgery. Tumors were staged using the tumor-node-metastasis classification system according to the International Union Against Cancer criteria. Ethical approval for the use of patient tissues was provided by the Ethics Committee of Shanghai Sixth People's Hospital (Shanghai, China). Informed consent was obtained from all patients. Data regarding patient age, sex, pathological grading, lymph node metastasis (LNM), tumor size and depth were collected followed the criteria proposed by International Union Against Cancer criteria (19). The follow-up period ranged between 5.3 and 48.8 months, and mean follow-up time was 32.1 months.

The three human ESCC cell lines ECA109, TE1 and KYSE30 were purchased from the China Academy of Science cell library (Beijing, China). Cells were cultured in RPMI-1640 medium (Gibco; Thermo Fisher Scientific, Inc., Waltham, MA, USA) supplemented with $10 \%$ fetal bovine serum (Gibco; Thermo Fisher Scientific, Inc.), penicillin (100 U/ml) and streptomycin $(100 \mu \mathrm{g} / \mathrm{ml})$ at $5 \% \mathrm{CO}_{2}$ and $37^{\circ} \mathrm{C}$. In cisplatin-related experiments, TE-1 cells were seeded onto 6-well cell culture cluster plates (Corning inc, Corning, NY, USA) at a concentration of $1 \times 10^{6}$ cells/well in $2 \mathrm{ml}$ culture medium. After being grown overnight, the cells were treated with cisplatin (cat. no., CAS 15663-27-1, Santa Cruz Biotechnology, Inc., Dallas, TX, USA) at various concentrations $(0-50 \mu \mathrm{M})$ for $48 \mathrm{~h}$.

Immunohistochemistry (IHC). IHC was performed following a previously described protocol (20). The primary antibodies used were anti-CCT8 (1:800; cat. no. sc-13891, Santa Cruz Biotechnology, Inc.) and anti-E-cadherin (1:200; cat. no. sc-71009, Santa Cruz Biotechnology, Inc.). IHC analyses (mouse anti-goat IgG-HRP: cat. no. sc-2354, 1:2,000; m-IgGк BP-HRP: cat. no. sc-516102, 1:2,000, Santa Cruz Biotechnology, Inc.) were performed as previously described (21). A staining index (SI) score of $\geq 6$ indicated high expression and an SI score of $\leq 4$ indicated low expression (21).

Western blot analysis. Western blot analysis was performed following a previously described protocol (22). Protein was obtained using a lysis buffer (1\% SDS, $10 \mathrm{mM}$ Tris- $\mathrm{HCl}, \mathrm{pH} 7.6$, $20 \mu \mathrm{g} / \mathrm{ml}$ aprotinin, $20 \mu \mathrm{g} / \mathrm{ml}$ leupeptin and $1 \mathrm{mM}$ AEBSF). Protein were separated on a $10 \%$ SDS-PAGE gel.Polyvinylidene fluoride (PVDF) membranes (Millipore, Inc., Billerica, MA, USA) were blotted with anti-CCT8 (cat. no. sc-13891, 1:500), anti-caspase-3 (cat. no. sc-166589, 1:1,000), anti-E-cadherin (cat. no. sc-71009, 1:1,000), anti-N-cadherin (cat. no. sc-53488, 1:1,000), anti-vimentin (cat. no. sc-66001, 1:1,000), $\alpha$-actin (cat. no. sc-32251, 1:1,000), $\beta$-tubulin (cat. no. sc-5274, 1:1,000) and anti-GAPDH (cat. no. sc-137179, 1:500; all Santa Cruz Biotechnology, Inc.). Secondary antibodies used as follows: mouse anti-goat IgG-HRP: cat. no. sc-2354, 1:1,000;
m-IgGк BP-HRP: cat. no. sc-516102, 1:1,000 (Santa Cruz Biotechnology, Inc.). The band density was measured with a computer-assisted image-analysis system (Adobe Systems, San Jose, CA, USA) and normalized against GAPDH level.

siRNA and transfection. Primer pairs for the CCT8 (NM_006585.2) siRNA expression vector had the following target sequences: 5'-CCUCCAUAAUGAGUAAACA-3', 5'-GGC UGUGUAUAGAAACAUA-3' and 5'-CUGGAGUUA AGGCC AAUGA-3'. These sequences are pertaining to pires2-egfp. The CCT8 siRNA vector $\left(1 \mu \mathrm{g} / 1 \times 10^{5}\right.$ cells $)$ and the non-specific vector $\left(2 \mu 1 / 1 \times 10^{5}\right.$ cells $)$ were transfected for $48 \mathrm{~h}$ using Lipofectamine 2000 (Invitrogen; Thermo Fisher Scientific, Inc.).

Cell proliferation, migration and invasion assays. Cell proliferation and migration were measured as previously described $(23,24)$. Migration assays were performed using the microliter scale radial monolayer migration assay. Tumor cells $\left(5 \times 10^{5}\right)$ were seeded in 6-well plates and grown to confluence overnight. Twenty-four hours after incubation with $0.1 \%$ bovine serum albumin (BSA, Gibco; Thermo Fisher Scientific, Inc.) or $10 \mu \mathrm{g} / \mathrm{ml}$ laminin (cat. no. sc-29012, Santa Cruz Biotechnology, Inc.) conditions a line was scratched within the confluent cell layer using the fine end of a 10-ml pipette tip (time 0). Cells were washed with phosphate-buffered saline (PBS, Procell Inc., Wuhan, China) and photographed under a 20X objective lens every $6 \mathrm{~h}$ using an inverted Leica phase-contrast microscope (Leica DFC 300 FX, Leica Microsystems, Inc., Wetzlar, Germany). The invasiveness of cells was determined using a Transwell assay on a 24 -well Transwell plate $(8-\mu \mathrm{m}$ pore size; Corning Inc., Corning, NY, USA). A total of $1 \times 10^{5}$ cells were plated in the top chamber. BD Matrigel ${ }^{\mathrm{TM}}$ Basement Membrane Matrix was added to the upper membrane and allowed to gel for $1 \mathrm{~h}$. Subsequently, $500 \mu 1$ medium containing chemotactic factor was placed in the lower chamber. Cells were plated in the upper chamber of quadruplicate wells at a density of $1 \times 10^{5} / \mathrm{ml}$ and incubated for $24 \mathrm{~h}$. Cells were then fixed with paraformaldehyde, stained with crystal violet (cat. no. sc-207460, Santa Cruz Biotechnology, Inc.), and counted immediately after staining.

Statistical analysis. The Predictive Analytics Software 18 software package (SPSS, Inc., Chicago, IL, USA) was used for statistical analyses. Data are presented as the means \pm standard deviations (SD) of 3 independent experiments. The association between CCT8 expression and patient clinicopathological features was calculated using the $\chi^{2}$ test. Kaplan-Meier methods were used to construct survival curves. Multivariate analysis was performed using the Cox proportional hazards model. One-way analysis of variance followed by Tukey's post hoc test was used to determine whether differences between groups were significant and $\mathrm{P}<0.05$ was considered to indicate a statistically significant difference.

\section{Results}

CCT8 expression is associated with the clinicopathological variables of patients with ESCC. CCT8 and E-cadherin expression in the 128 patients with ESCC was assessed using IHC. CCT8 expression (Fig. 1) and its association 
Table I. CCT8 expression and clinicopathological parameters in 128 ESCC specimens.

\begin{tabular}{|c|c|c|c|c|c|}
\hline \multirow[b]{2}{*}{ Parameters } & \multirow[b]{2}{*}{ Total } & \multicolumn{2}{|c|}{ CCT8 } & \multirow[b]{2}{*}{ P-values } & \multirow[b]{2}{*}{$\chi^{2}$} \\
\hline & & Score $\leq 4, \mathrm{n}(\%)$ & Score $\geq 6, \mathrm{n}(\%)$ & & \\
\hline \multicolumn{6}{|l|}{ Age, years } \\
\hline$<60$ & 51 & $26(51.0)$ & $25(49.0)$ & 0.406 & 0.691 \\
\hline$\geq 60$ & 77 & $45(58.4)$ & $32(41.6)$ & & \\
\hline \multicolumn{6}{|l|}{ Sex } \\
\hline Female & 41 & $21(51.2)$ & $20(48.8)$ & 0.507 & 0.441 \\
\hline Male & 87 & $50(57.5)$ & $37(42.5)$ & & \\
\hline \multicolumn{6}{|c|}{ Pathological grading } \\
\hline Well & 28 & $25(89.3)$ & $3(10.7)$ & $<0.001^{\mathrm{a}}$ & 41.744 \\
\hline Moderate & 49 & $35(71.4)$ & 14 (28.6) & & \\
\hline Poor & 51 & $11(21.6)$ & $40(78.4)$ & & \\
\hline \multicolumn{6}{|c|}{ Lymph node metastasis } \\
\hline Presence & 32 & $9(28.1)$ & $23(71.9)$ & $<0.001^{\mathrm{a}}$ & 12.915 \\
\hline Absence & 96 & $62(64.6)$ & $34(35.4)$ & & \\
\hline \multicolumn{6}{|c|}{ Tumor size, cm } \\
\hline$<5$ & 99 & $55(55.6)$ & $44(44.4)$ & 0.971 & 0.001 \\
\hline$\geq 5$ & 29 & $16(55.2)$ & $13(44.8)$ & & \\
\hline \multicolumn{6}{|c|}{ Tumor depth } \\
\hline $\mathrm{T} 1$ & 45 & $33(73.3)$ & $12(26.7)$ & $0.011^{\mathrm{a}}$ & 9.004 \\
\hline $\mathrm{T} 2$ & 34 & $16(47.1)$ & $18(52.9)$ & & \\
\hline $\mathrm{T} 3$ & 49 & $22(44.9)$ & $27(55.1)$ & & \\
\hline \multicolumn{6}{|l|}{ E-cadherin } \\
\hline Low & 44 & $35(79.5)$ & $9(20.5)$ & $<0.001^{\mathrm{a}}$ & 15.735 \\
\hline High & 84 & $36(42.9)$ & $48(57.1)$ & & \\
\hline
\end{tabular}

Statistical analyses were performed using the $\chi^{2}$ test. ${ }^{\mathrm{a}} \mathrm{P}<0.05$. CCT8, chaperonin containing T-complex protein 1 subunit 8 ; ESCC, esophageal squamous cell carcinoma.
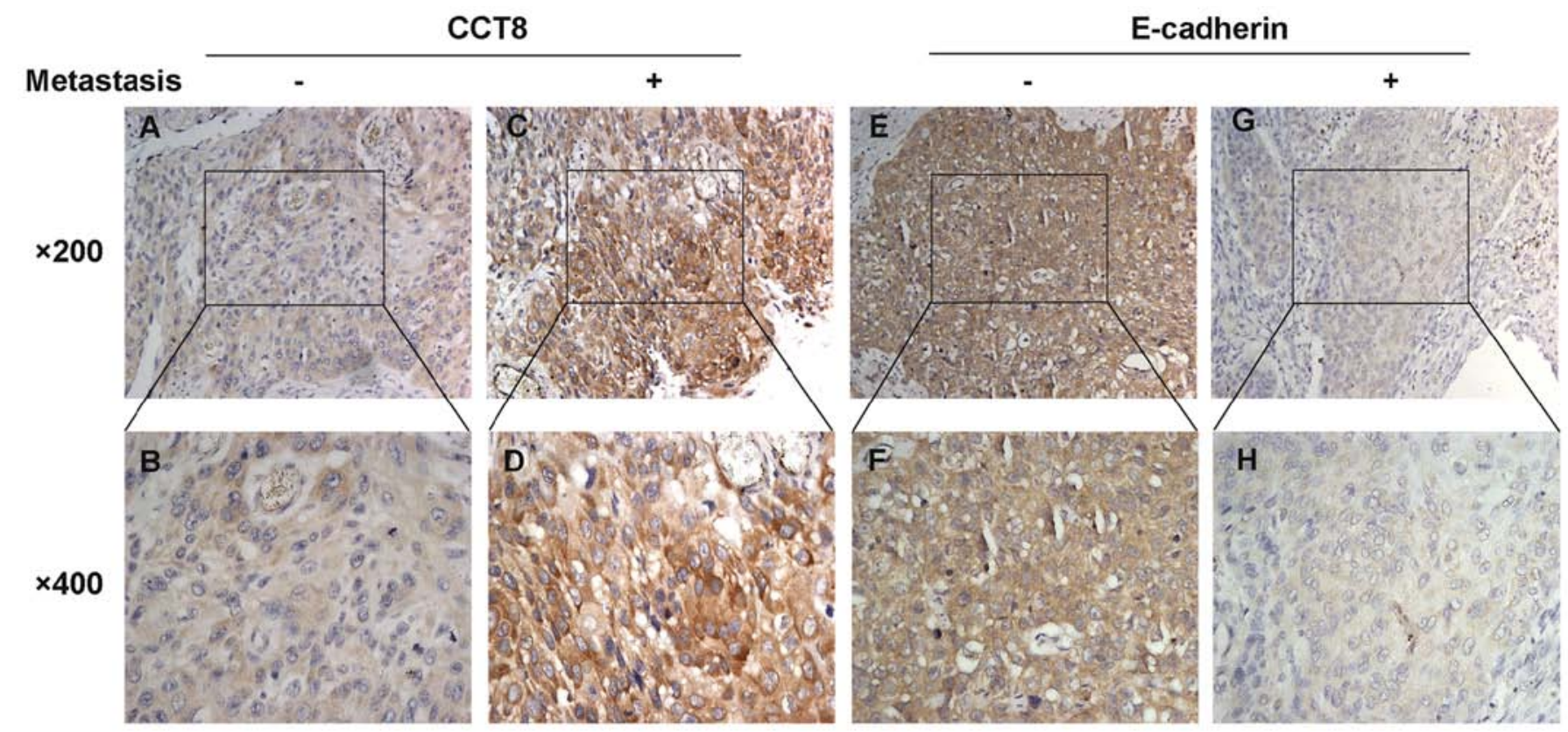

Figure 1. Immunohistochemical analysis of CCT8 and E-cadherin expression in 128 ESCC tissues. (A B, E and F) ESCC tissues with no LNM exhibited low CCT8 and high E-cadherin expression. (C, D, G and H) ESCC tissues with LNM exhibited high CCT8 and low E-cadherin expression. LNM, lymph node metastasis; ESCC, esophageal squamous cell carcinoma; CCT8, chaperonin containing T-complex protein 1 subunit 8. 
Table II. Survival status and clinicopathological parameters in 128 ESCC specimens.

\begin{tabular}{|c|c|c|c|c|c|}
\hline & \multirow[b]{2}{*}{ Total } & \multicolumn{2}{|c|}{ Survival status } & \multirow[b]{2}{*}{ P-values } & \multirow[b]{2}{*}{$\chi^{2}$} \\
\hline & & Succumbed, n (\%) & Alive, $\mathrm{n}(\%)$ & & \\
\hline \multicolumn{6}{|l|}{ Age (years) } \\
\hline$<60$ & 51 & $23(45.1)$ & $28(54.9)$ & \multirow[t]{2}{*}{0.743} & \multirow[t]{2}{*}{0.107} \\
\hline$\geq 60$ & 77 & $37(48.1)$ & $40(51.9)$ & & \\
\hline \multicolumn{6}{|l|}{ Sex } \\
\hline Female & 41 & $17(41.5)$ & $24(58.5)$ & \multirow[t]{2}{*}{0.400} & \multirow[t]{2}{*}{0.709} \\
\hline Male & 87 & $43(49.4)$ & $44(50.6)$ & & \\
\hline \multicolumn{6}{|c|}{$\begin{array}{l}\text { Pathological } \\
\text { grading }\end{array}$} \\
\hline Well & 28 & $5(17.9)$ & $23(82.1)$ & \multirow[t]{3}{*}{$<0.001^{\mathrm{a}}$} & \multirow[t]{3}{*}{22.275} \\
\hline Moderate & 49 & $19(38.8)$ & $30(61.2)$ & & \\
\hline Poor & 51 & $36(70.6)$ & $15(29.4)$ & & \\
\hline \multicolumn{6}{|c|}{ Lymph node metastasis } \\
\hline Presence & 32 & $28(87.5)$ & $4(12.5)$ & \multirow[t]{2}{*}{$0.041^{\mathrm{a}}$} & \multirow[t]{2}{*}{6.372} \\
\hline Absence & 96 & $32(33.3)$ & 64 (66.7) & & \\
\hline \multicolumn{6}{|c|}{ Tumor size, cm } \\
\hline$<5$ & 99 & $45(45.5)$ & $54(54.5)$ & \multirow[t]{2}{*}{0.552} & \multirow[t]{2}{*}{0.354} \\
\hline$\geq 5$ & 29 & $15(53.8)$ & $14(46.2)$ & & \\
\hline \multicolumn{6}{|c|}{ Tumor depth } \\
\hline $\mathrm{T} 1$ & 45 & $5(11.1)$ & $40(88.9)$ & \multirow[t]{3}{*}{$0.035^{\mathrm{a}}$} & \multirow[t]{3}{*}{7.952} \\
\hline $\mathrm{T} 2$ & 34 & $19(55.9)$ & $15(44.1)$ & & \\
\hline $\mathrm{T} 3$ & 49 & $36(73.5)$ & $13(26.5)$ & & \\
\hline \multicolumn{6}{|l|}{ ССТ8 } \\
\hline Score $<4$ & 71 & $22(31.0)$ & $49(69.0)$ & \multirow[t]{2}{*}{$<0.001^{\mathrm{a}}$} & \multirow[t]{2}{*}{16.164} \\
\hline Score $\geq 6$ & 57 & $38(66.7)$ & $19(33.3)$ & & \\
\hline \multicolumn{6}{|c|}{ E-cadherin expression } \\
\hline Low & 44 & $13(29.5)$ & $31(70.50)$ & \multirow[t]{2}{*}{$0.004^{\mathrm{a}}$} & \multirow[t]{2}{*}{8.086} \\
\hline High & 84 & $47(56.0)$ & $37(44.0)$ & & \\
\hline
\end{tabular}

Statistical analyses were performed using the $\chi^{2}$ test. ${ }^{\mathrm{a}} \mathrm{P}<0.05$. CCT 8 , chaperonin containing T-complex protein 1 subunit 8 ; ESCC, esophageal squamous cell carcinoma.

with the clinicopathological variables of ESCC is presented in Tables I and II. Following a previously described protocol (22), patients were divided into two groups: One group contained patients exhibiting high CCT8 expression (score $\geq 6$ ) and one group contained patients exhibiting low CCT8 expression (score $\leq 4$ ). The results demonstrated that CCT8 expression was associated with pathological grading $(\mathrm{P}<0.001)$, tumor depth $(\mathrm{P}<0.011)$ and LNM $(\mathrm{P}<0.001)$; however, it was not associated with other prognostic factors, including tumor size (Table I). These results indicate that there is an association between CCT8 expression and the degree of ESCC malignancy. A total of 38 out of 57 (66.7\%) patients in the high CCT8 expression group succumbed compared with only 22 of $71(31.0 \%)$ patients in the low expression group $(\mathrm{P}<0.001$; Table II). Furhthermore, patients with poor pathological grading $(\mathrm{P}<0.001)$, LNM $(\mathrm{P}=0.041)$, greater tumor depth $(\mathrm{P}=0.035)$ and high $\mathrm{E}$-cadherin expression $(\mathrm{P}=0.004)$ exhibited significantly lower rates of survival (Table II).

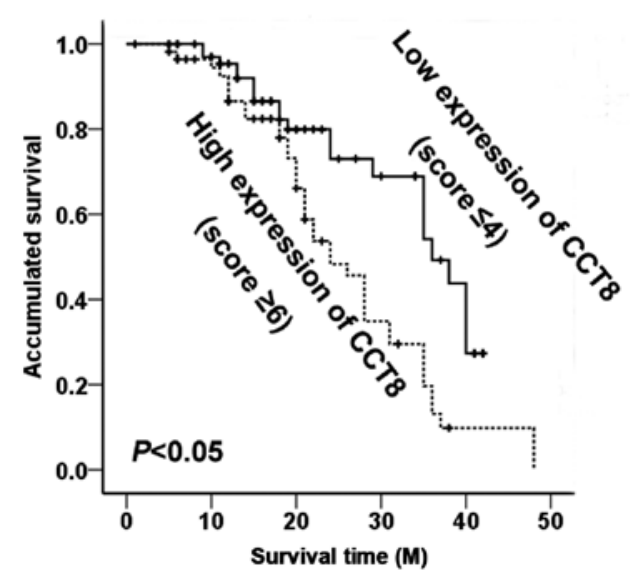

Figure 2. Association between cumulative survival and CCT8 expression Based on the level of CCT8 expression, the 128 patients were divided into two groups based on the level of CCT8 expression. Kaplan-Meier analysis indicated that patients exhibiting high expression of CCT8 had poorer overall survival rates. $\mathrm{M}$, months; $\mathrm{CCT} 8$, chaperonin containing T-complex protein 1 subunit 8 . 


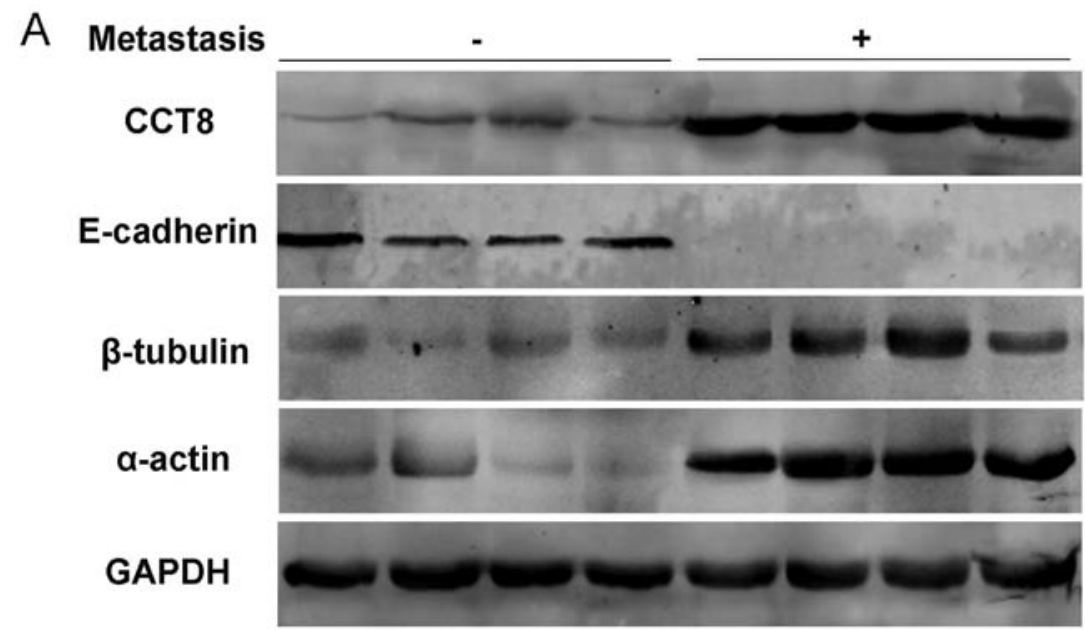

B

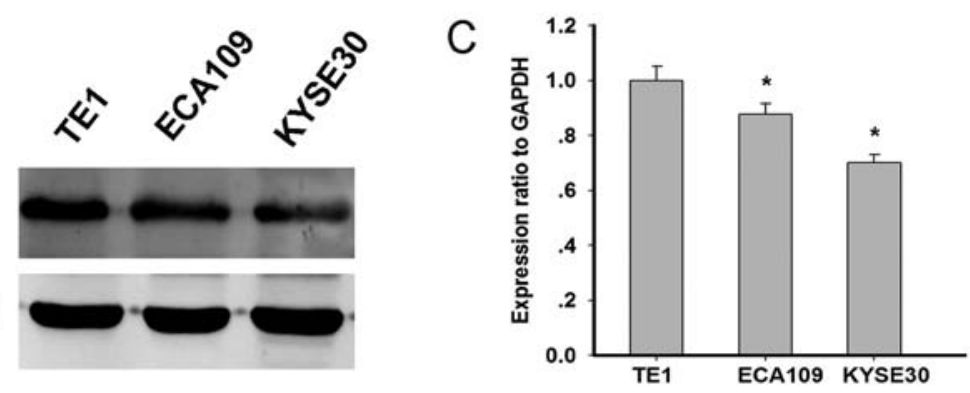

Figure 3. CCT8 expression in human ESCC cells. (A) Expression of CCT8, E-cadherin, $\alpha$-actin and $\beta$-tubulin in eight ESCC samples. (B) Western blot analysis of CCT8 expression in the three human ESCC cell lines TE-1, ECA109 and KYSE30. GAPDH was used as a loading control. (C) Quantification of the western blot analysis results indicated that TE-1 cell lines exhibited the highest expression of CCT8 among the tumor cell lines. "P<0.05 vs. TE-1. ESCC, esophageal squamous cell carcinoma; CCT8, chaperonin containing T-complex protein 1 subunit 8.

Kaplan-Meier survival curves were then constructed to determine the survival rates of patients in the high CCT8 expression group compared with those in the low CCT8 expression group. The results indicated that the survival time of patients in the high CCT8 expression group was significantly lower than that of patients in the low CCT8 expression group $(\mathrm{P}<0.05$; Fig. 2). A multivariate Cox proportional hazards model was then used to predict patient survival. The results demonstrated that tumor pathological grade, LNM, tumor depth, CCT8 and E-cadherin expression were significant predictors of patient survival $(\mathrm{P}<0.05$; Table III).

CCT8 expression is increased in highly metastatic ESCC tissues. As CCT8 expression is associated with LNM in ESCC, it was hypothesized that it may also be associated with the migration of ESCC cells. Therefore, the expression of CCT8 in ESCC tissues was evaluated (Fig. 3A). CCT8 expression was high in tissues taken from patients with LNM and low in tissues from patients without LNM. In addition, CCT8 expression was measured in the TE-1, ECA109 and KYSE30 ESCC cell lines (Fig. 3B). Quantification of the results indicated that TE-1 cells exhibited the highest level of CCT8 expression among the ESCC cell lines (Fig. 3C); therefore, TE-1 cells were used in subsequent experiments.

Actively migrating cells highly express CCT8. It was hypothesized that CCT8 expression is associated with tumor migration. A monolayer wound healing assay was used to assess the migration rate of cells plated onto the nonpermissive substrate
Table III. Contribution of various potential prognostic factors to survival assessed using Cox regression analysis in 128 ESCC specimens.

\begin{tabular}{lccc}
\hline & $\begin{array}{c}\text { Relative } \\
\text { ratio }\end{array}$ & $\begin{array}{c}95 \% \text { Confidence } \\
\text { interval }\end{array}$ & P-values \\
\hline Age (years) & 1.318 & $0.777-2.236$ & 0.305 \\
Sex & 0.933 & $0.529-1.644$ & 0.809 \\
Tumor size & 0.786 & $0.433-1.427$ & 0.430 \\
Lymph node & 1.724 & $1.043-3.337$ & $0.043^{\mathrm{a}}$ \\
metastasis & & & \\
Pathological & 2.308 & $1.557-3.420$ & $<0.001^{\mathrm{a}}$ \\
grading & & & \\
Tumor depth & 1.824 & $0.961-3.143$ & $0.038^{\mathrm{a}}$ \\
CCT8 & 2.235 & $1.306-3.826$ & $0.003^{\mathrm{a}}$ \\
E-cadherin & 2.592 & $1.392-4.827$ & $0.003^{\mathrm{a}}$ \\
\hline
\end{tabular}

Statistical analyses were performed using the Cox test. ${ }^{\mathrm{a}} \mathrm{P}<0.05$. CCT8, CCT8, chaperonin containing T-complex protein 1 subunit 8; ESCC, esophageal squamous cell carcinoma.

BSA and the migration-stimulating substrate, laminin $(24,25)$. It was demonstrated that laminin significantly increased the migration of TE-1 compared with those cells treated with BSA ( $\mathrm{P}<0.05$; Fig. $4 \mathrm{~A})$. To determine whether the migratory 


\section{A a \\ LN}
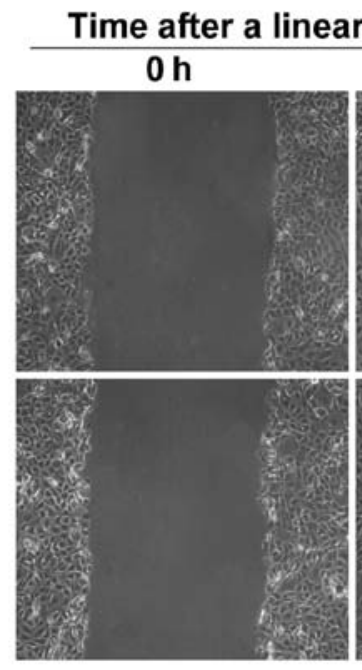

B

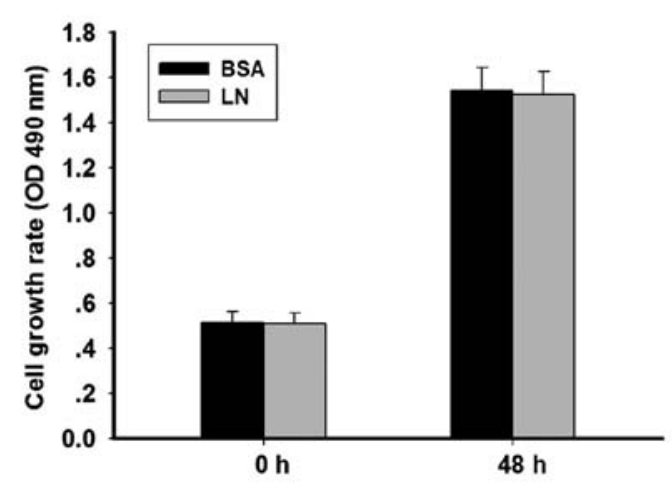

b

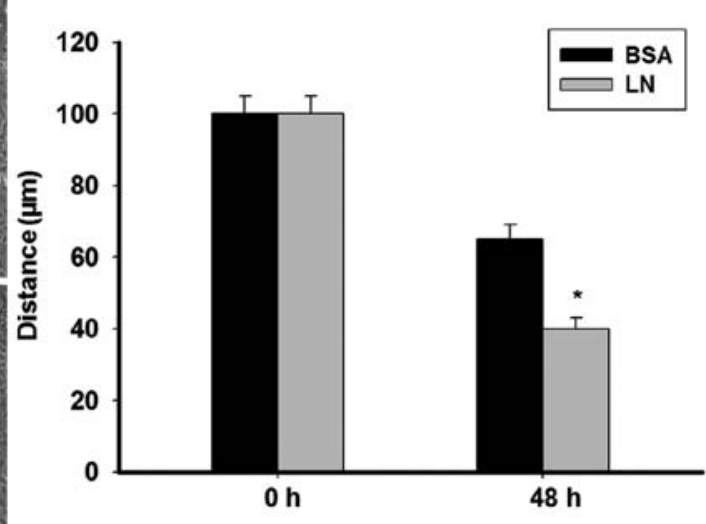

C

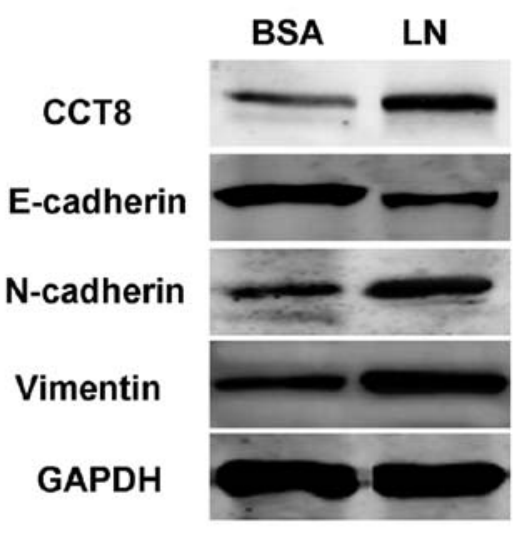

Figure 4. Actively migrating cells express high levels of CCT8. (A) The results of the wound healing assay demonstrated that LN stimulates the migration of TE-1 cells. * $\mathrm{P}<0.05$ vs. BSA. (B) LN did not alter TE-1 cell proliferation, as determined by the Cell Counting Kit-8. (C) Western blot analysis of CCT8, E-cadherin, N-cadherin and vimentin expression in TE-1 cells cultured on BSA- or LN-coated dishes. The results are presented as the mean \pm standard error of the mean $(\mathrm{n}=3) .{ }^{*} \mathrm{P}<0.05$ vs. dense. LN, laminin; BSA, bovine serum albumin; CCT8, chaperonin containing T-complex protein 1 subunit 8 ; OD, optical density.

phenotype affected cell proliferation, a Cell Counting Kit-8 (Dojindo Molecular Technologies, Inc., Kumamato, Japan) was used and the cell growth rate was determined. The results demonstrated that laminin stimulated TE-1 cell migration in vitro but did not affect cell proliferation (Fig. 4B). When TE-1 cells were plated on laminin, CCT8 expression increased markedly, as did the expression of $\mathrm{N}$-cadherin and vimentin; by contrast, the expression of E-cadherin markedly decreased (Fig. 4C). These results suggest that CCT8 may serve a role in the migration of ESCC cells.

CCT8 promotes ESCC cell migration and invasion in vitro by regulating $\alpha$-actin and $\beta$-tubulin. To assess the function of CCT8 in ESCC cells, CCT8 expression was knocked down and western blotting was used to verify this loss of expression (Fig. 5A). Three target sites were selected to knockdown CCT8 expression. The most significant decrease in CCT8 expression was following CCT8 knockdown with si\#1 and si\#2 $(\mathrm{P}<0.01$ and $\mathrm{P}<0.05$, respectively; Fig. $5 \mathrm{~B}$ ); therefore, these were used in subsequent studies. CCT8 knockdown also significantly inhibited the wound healing process $(\mathrm{P}<0.05$; Fig. $5 \mathrm{C}$ and $\mathrm{D})$ and significantly decreased the invasiveness of TE-1 cells (Fig. 5E and F). CCT8 knockdown also markedly increased the expression of E-cadherin and decreased the expression of
$\mathrm{N}$-cadherin and vimentin (Fig. 5G). Furthermore, expression of the apoptosis marker, cleaved caspase-3, was increased. It has been reported that CCT 8 interacts with $\alpha$-actin and $\beta$-tubulin, which can stimulate cell motility (26). The results of western blotting demonstrated that $\alpha$-actin and $\beta$-tubulin were markedly downregulated following CCT8 knockdown (Fig. 5G). These observations suggest that CCT8 facilitates cell motility and is associated with the regulation of $\alpha$-actin and $\beta$-tubulin.

CCT8 expression is closely associated with cisplatin-treated $T E-1$ cells. Cisplatin is a chemotherapy drug widely used to treat different types of cancer, including ESCC (27); therefore, it was determined whether cisplatin downregulates CCT8 expression in ESCC. TE-1 cells treated with cisplatin for $48 \mathrm{~h}$ exhibited a dose-dependent decrease in CCT8 expression and a dose-dependent increase in cleaved caspase-3 expression (Fig. 6A). $\alpha$-actin and $\beta$-tubulin expression also decreased in a dose-dependent manner (Fig. 6A). Cisplatin induced the upregulation of E-cadherin and decreased the expression of $\mathrm{N}$-cadherin, vimentin, $\alpha$-actin and $\beta$-tubulin. These effects were enhanced following CCT8 knockdown (Fig. 6B). These data demonstrate that CCT8 knockdown significantly increases the apoptosis of TE-1 cells and enhanced the effects of cisplatin on such cells. 


\section{A a \\ ССТ8 \\ GAPDH}

B a
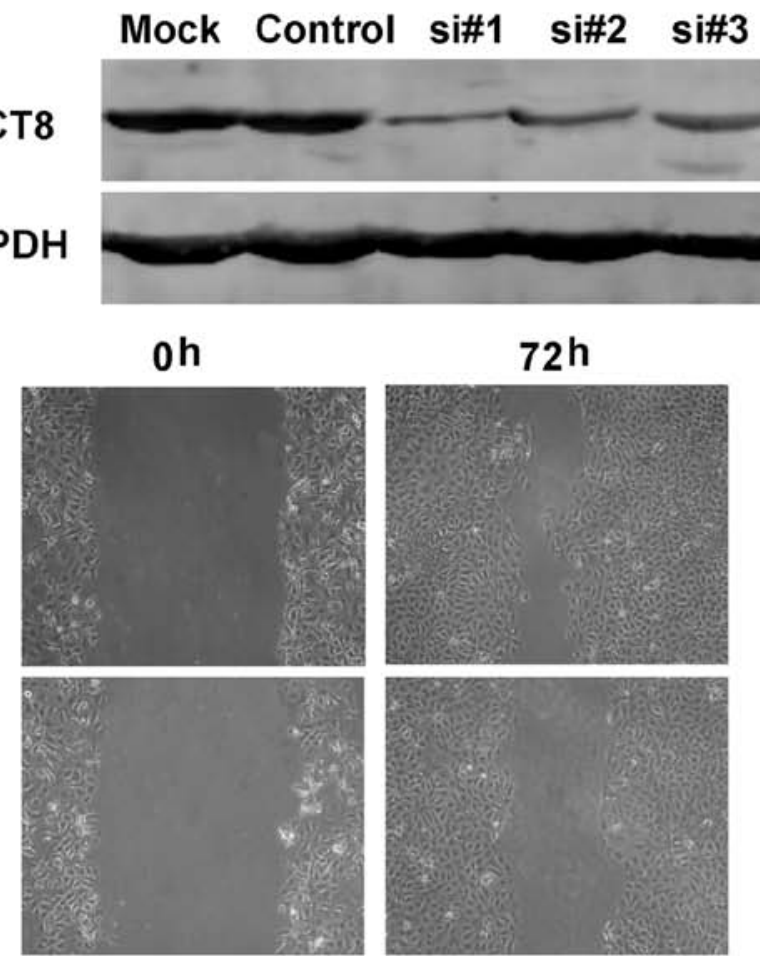

Control

si\#1

i\#2
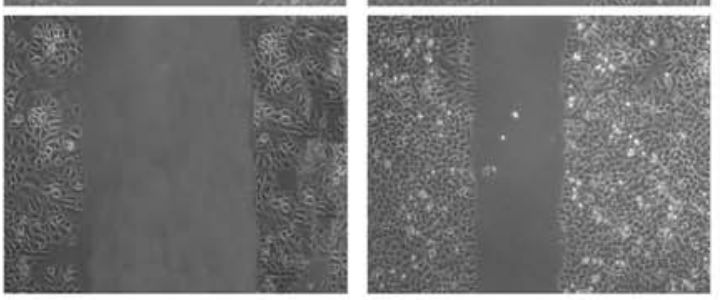

$\mathrm{B} b$

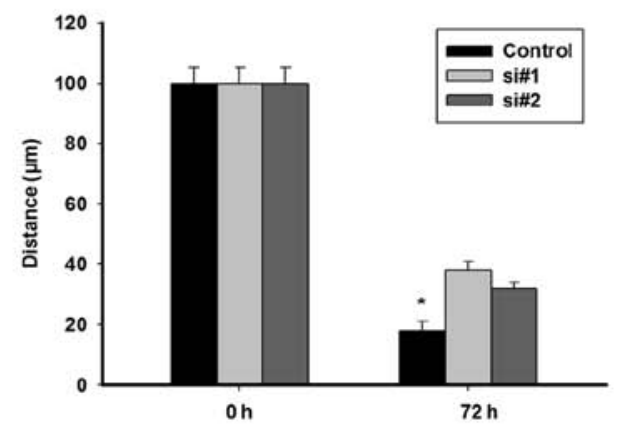

$\mathrm{C} b$

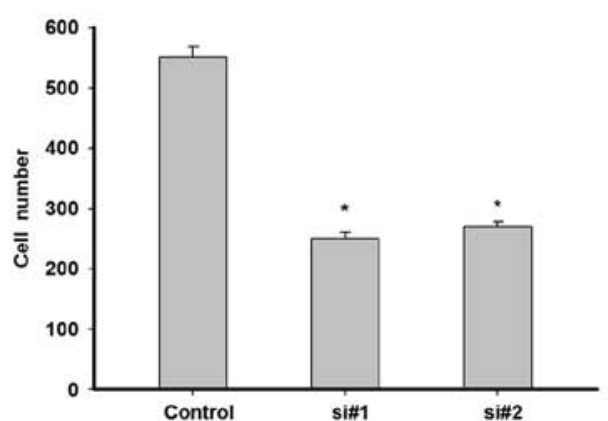

$72 \mathrm{~h}$
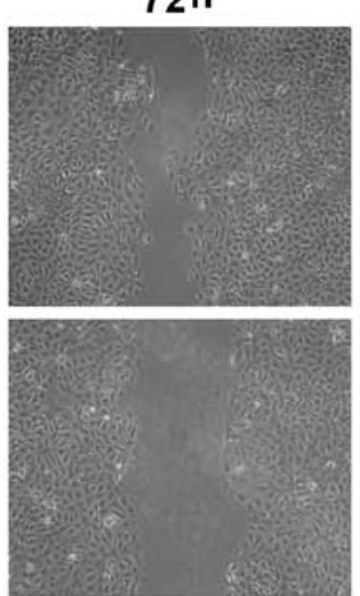

D

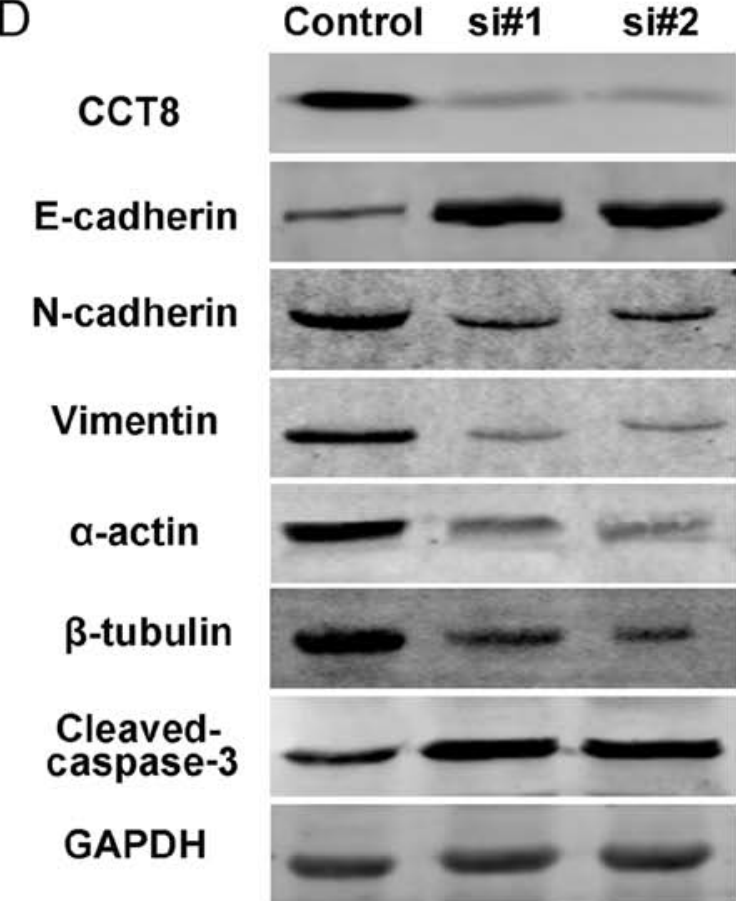

Figure 5. CCT8 promotes ESCC cell migration and invasion in vitro by regulating $\alpha$-actin and $\beta$-tubulin expression. (A) Western blot analysis of CCT8 in control cells and following transfection with mock, si\#1, si\#2 and si\#3 CCT8. (B) Quantification of CCT8 following transfection. "P<0.05 and ${ }^{* *} \mathrm{P}<0.01$ vs. control. (C) Wound healing assays with control, si\#1 and si\#2 cells (magnification, x200). (D) Quantification of wound healing assay results. "P<0.05 vs. $0 \mathrm{~h}$. (E) Transfection with siCCT8 inhibited the invasiveness of cells (magnification, x20). (F) The number of cells that invaded through the membrane was counted in 10 fields. "P $<0.05$ vs. control. (G) Western blot analysis assessing the expression of CCT 8 , E-cadherin, N-cadherin, vimentin, $\alpha$-actin, $\beta$-tubulin and cleaved caspase-3. GAPDH was used as a loading control. The results are presented as the mean \pm standard deviation. si, small interfering; CCT8, chaperonin containing T-complex protein 1 subunit 8; ESCC, esophageal squamous cell carcinoma. 


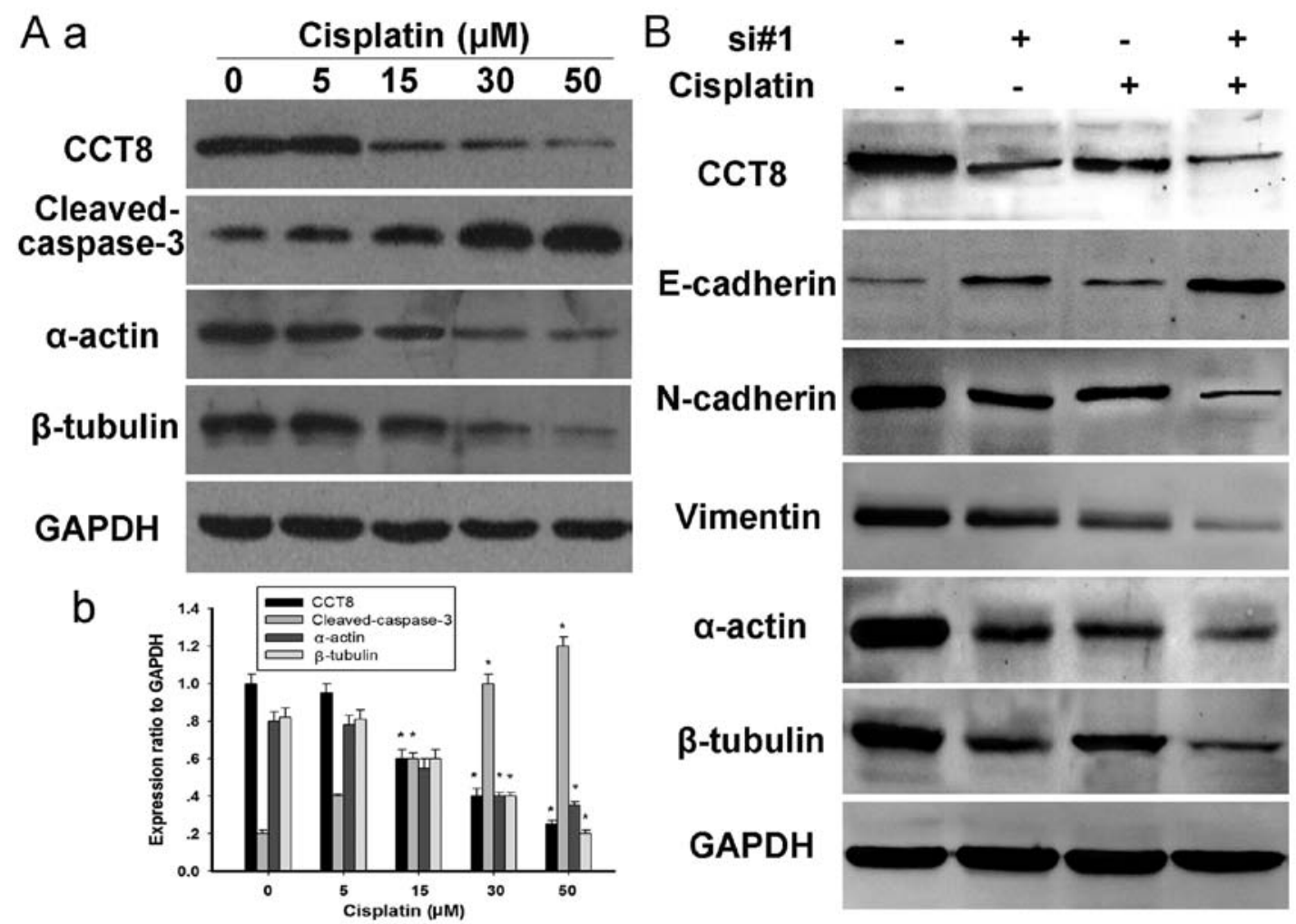

Figure 6. Cisplatin treatment inhibits CCT8 expression in a dose-dependent manner in TE-1 cells. (A) Western blot analysis indicated the dose-dependent effect of cisplatin on CCT8, $\alpha$-actin and $\beta$-tubulin expression in TE- 1 cells following treatment with different concentrations of cisplatin $(0,5,15,30$ and $50 \mu \mathrm{M}$ ) for $48 \mathrm{~h}$. The bar chart demonstrated the ratio of CCT8, cleaved caspase-3, $\alpha$-actin and $\beta$-tubulin protein to GAPDH by densitometry. (B) Western blot analysis indicated the effects of cisplatin on CCT8, E-cadherin, N-cadherin, vimentin, $\alpha$-actin and $\beta$-tubulin expressions in TE- 1 cells, which were transfected with control-siRNA or CCT8-siRNA for $24 \mathrm{~h}$, and then subjected to treatment with or without cisplatin for $48 \mathrm{~h}$, respectively. Data are presented as the mean \pm standard error of the mean. ${ }^{~} \mathrm{P}<0.05$ vs. respective controls. si, small interfering; CCT8, chaperonin containing T-complex protein 1 subunit 8.

\section{Discussion}

ESCC is a common malignancy affecting people across the world. LNM is an important prognostic indicator for patients with ESCC and may influence the treatments such patients receive. In the present study, CCT8 expression in 128 patients with ESCC was assessed and statistical analysis demonstrated that there was an association between CCT8 expression and LNM. Furthermore, the survival rates of patients with high CCT8 expression were significantly decreased compared with those exhibiting low CCT8 expression. Therefore, CCT8 expression may be a useful prognostic factor in determining the survival of patients with ESCC.

$\mathrm{CCT}$ is a cytosolic chaperonin that interacts with various cellular proteins (28). CCT8 is a microtubule-associated protein (29) and Rademacher et al (30) demonstrated that CCT is involved in cytoskeletal rearrangements. Consequently, it was hypothesized that CCT8 may be associated with tumor cell migration. Cell migration serves an important role in several physiological processes, including wound healing, tissue morphogenesis and metastasis (31). siRNAs were used to knockdown CCT8 expression and identify whether decreased CCT8 expression influences the motility of TE- 1 cells. The results indicated that, following CCT8 knockdown, TE-1 cells exhibited decreased migratory and invasive abilities compared with normal cells. CCT8 knockdown also altered the expression of epithelial-Mesenchymal transition markers, including E-cadherin, $\mathrm{N}$-cadherin and vimentin.
Previous studies have demonstrated that CCT8 is associated with cell proliferation in B-cell non-Hodgkin's lymphoma (16), glioma (17) and hepatocellular carcinoma (18). The results of the current study indicated that CCT8 knockdown induces cell death. Combination treatment of cisplatin with siCCT8 decreased the ability of ESCC cells to migrate and invade, thus inducing the death of ESCC cells. These data indicate that CCT8 serves an essential role in the migration and invasion of ESCC.

The microtubule, which is composed of $\alpha$-actin and $\beta$-tubulin (26) is essential to cellular activities including cell division, polarization and migration (32). Proteins associated with $\alpha$-actin and $\beta$-tubulin serve a key role in particular cellular processes, and CCT8 is closely associated with $\alpha$-actin and $\beta$-tubulin (33-35). The results of the current study demonstrated that CCT8 knockdown decreased $\alpha$-actin and $\beta$-tubulin expression in ESCC cells. It has been suggested that $\alpha$-actin and $\beta$-tubulin act as natural substrates for CCT, indicating that the chaperone may serve an important role in maintaining the integrity of the cytoskeleton (36). Hence, CCT gene mutations weaken $\alpha$-actin- and $\beta$-tubulin-dependent structures (37). However, the precise mechanism by which CCT8 stimulates the activity of $\alpha$-actin and $\beta$-tubulin remains unknown. It has been demonstrated that CCT8 interacts with $\alpha$-actin and $\beta$-tubulin (22) and the results of the current study demonstrated that CCT8 knockdown decreased the expression of $\alpha$-actin and $\beta$-tubulin. It was suggested that the combination of CCT8 and $\alpha$-actin or $\beta$-tubulin induced changes in the expression of $\alpha$-actin 
and $\beta$-tubulin. This indicates that CCT8 forms a complex with $\alpha$-actin and $\beta$-tubulin and subsequently stimulates the migration of ESCC. The CCT complex regulates many proteins; however, it is possible that the decreased migration and invasion of ESCC cells observed following CCT8 knockdown were due to the impaired function of other proteins as well as $\alpha$-actin and $\beta$-tubulin. Further studies are required to investigate whether this is the case. Furthermore, the response of cells to cisplatin treatment following CCT8 knockdown indicated that CCT8 siRNA induces the death of ESCC cells due to the combined regulation of $\alpha$-actin and $\beta$-tubulin by CCT 8 and cisplatin. This indicates that CCT8 promotes the migration and invasion of ESCC cells by activating the expression of $\alpha$-actin and $\beta$-tubulin.

The two primary predictors for the survival of patients with malignant tumors are uncontrolled growth and invasiveness. However, no association between CCT8 expression and tumor size was observed in the current study. Furthermore, the cohort size was small and studies involving a larger number of patients are required to ensure that these results can be replicated. Future studies, including randomized controlled trials, are required to validate the results of the current study.

In conclusion, the present study demonstrated that CCT8 enhanced the migration and invasion of ESCC cells, indicating that ectopic CCT8 expression contributes to the highly aggressive nature of ESCC. This stimulatory effect of CCT8 on cell migration and invasion was accomplished by increasing $\alpha$-actin and $\beta$-tubulin expression. These results demonstrate that CCT8 is a critical factor in the migration and invasion of ESCC cells. Given that CCT8 expression is widely upregulated in ESCC, CCT8 silencing may be a promising method of preventing and treating the metastasis of ESCC cells.

\section{Acknowledgements}

The authors would like to thank LetPub (www.letpub.com) for its linguistic assistance during the preparation of this manuscript.

\section{Funding}

$\mathrm{XY}$ is the recipient of a grant of Shanghai Jiao Tong University Affiliated Sixth People's Hospital (contract grant number: ynlc201601). JF is the recipient of a grant of Shanghai Shenkang Hospital Development Center Funds for Three Year Action Plan for Promoting Clinical Skills and Clinical Innovation Capacity in Municipal Hospitals (16CR3112B).

\section{Availability of data and materials}

All data generated or analyzed during this study are included in this published article.

\section{Authors' contributions}

XiaojingY, HR and JF conceived and designed the study. XiaojingY, HR, YSh, YSu, LZ, HL, XZ, XinmiaoY and WY performed the experiments. XiaojingY and HR wrote the manuscript. XiaojingY and JF reviewed and edited the manuscript. All authors have read and approved the manuscript.

\section{Ethics approval and consent to participate}

Ethical approval for the use of patient tissues was provided by the Ethics Committee of Shanghai Sixth People's Hospital (Shanghai, China). Informed consent was obtained from all patients.

\section{Consent for publication}

Not applicable.

\section{Competing Interests}

The authors confirm that they have no conflicts of interest.

\section{References}

1. Feng JF, Yang X, Chen S, Zhao Q and Chen QX: Prognostic value of plasma D-dimer in patients with resectable esophageal squamous cell carcinoma in China. J Cancer 7: 1663-1667, 2016.

2. Tang S, Gao L, Bi Q, Xu G, Wang S, Zhao G, Chen Z, Zheng X, Pan Y,Zhao L, et al: SDR9C7 promotes lymph node metastases in patients with esophageal squamous cell carcinoma. PLoS One 8: e52184, 2013.

3. Kudo-Saito C, Shirako H, Takeuchi T and Kawakami Y: Cancer metastasis is accelerated through immunosuppression during Snailinduced EMT of cancer cells. Cancer Cell 15: 195-206, 2009.

4. Huang SD, Yuan Y, Zhuang CW, Li BL, Gong DJ, Wang SG, Zeng ZY and Cheng HZ: MicroRNA-98 and microRNA-214 post-transcriptionally regulate enhancer of zeste homolog 2 and inhibit migration and invasion in human esophageal squamous cell carcinoma. Mol Cancer 11: 51, 2012.

5. Gholipour M,Islami F, Roshandel G, Khoshnia M, Badakhshan A, Moradi A and Malekzadeh R: Esophageal cancer in Golestan Province, Iran: A review of genetic susceptibility and environmental risk factors. Middle East J Dig Dis 8: 249-266, 2016.

6. Hynes G, Celis JE, Lewis VA, Carne A, U S, Lauridsen JB and Willison KR: Analysis of chaperonin-containing TCP-1 subunits in the human keratinocyte two-dimensional protein database: Further characterisation of antibodies to individual subunits. Electrophoresis 17: 1720-1727, 1996.

7. Saegusa K, Sato M, Sato K, Nakajima-Shimada J, Harada A and Sato K: Caenorhabditis elegans chaperonin CCT/TRiC is required for actin and tubulin biogenesis and microvillus formation in intestinal epithelial cells. Mol Biol Cell 25: 3095-3104, 2014.

8. Fares MA and Wolfe KH: Positive selection and subfunctionalization of duplicated CCT chaperonin subunits. Mol Biol Evol 20: 1588-1597, 2003.

9. Grantham J, Brackley KI and Willison KR: Substantial CCT activity is required for cell cycle progression and cytoskeletal organization in mammalian cells. Exp Cell Res 312: 2309-2324, 2006.

10. Brackley KI and Grantham J: Subunits of the chaperonin CCT interact with F-actin and influence cell shape and cytoskeletal assembly. Exp Cell Res 316: 543-553, 2010.

11. Brackley KI and Grantham J: Activities of the chaperonin containing TCP-1 (CCT): Implications for cell cycle progression and cytoskeletal organisation. Cell Stress Chaperones 14: 23-31, 2009.

12. Lin YF, Lee YF and Liang PH: Targeting $\beta$-tubulin:CCT- $\beta$ complexes incurs Hsp90- and VCP-related protein degradation and induces ER stress-associated apoptosis by triggering capacitative $\mathrm{Ca}^{2+}$ entry, mitochondrial perturbation and caspase overactivation. Cell Death Dis 3: e434, 2012.

13. Yokota S, Yamamoto Y, Shimizu K, Momoi H, Kamikawa T, Yamaoka Y, Yanagi H, Yura $\mathrm{T}$ and Kubota H: Increased expression of cytosolic chaperonin CCT in human hepatocellular and colonic carcinoma. Cell Stress Chaperones 6: 345-350, 2001.

14. Yokota S, Yanagi H, Yura T and Kubota H: Cytosolic chaperonincontaining t-complex polypeptide 1 changes the content of a particular subunit species concomitant with substrate binding and folding activities during the cell cycle. Eur J Biochem 268: 46644673, 2001.

15. Guest ST, Kratche ZR, Bollig-Fischer A, Haddad R and Ethier SP: Two members of the TRiC chaperonin complex, CCT2 and TCP1 are essential for survival of breast cancer cells and are linked to driving oncogenes. Exp Cell Res 332: 223-235, 2015. 
16. Yin $\mathrm{H}$, Miao $\mathrm{X}, \mathrm{Wu} \mathrm{Y}$, Wei $\mathrm{Y}$, Zong $\mathrm{G}$, Yang S, Chen $\mathrm{X}$ Zheng G, Zhu X, Guo Y, et al: The role of the Chaperonin containing t-complex polypeptide 1 , subunit 8 (CCT8) in B-cell non-Hodgkin's lymphoma. Leuk Res 45: 59-67, 2016.

17. Qiu X, He X, Huang Q, Liu X, Sun G, Guo J, Yuan D, Yang L, Ban N, Fan S, et al: Overexpression of CCT8 and its significance for tumor cell proliferation, migration and invasion in glioma. Pathol Res Pract 211: 717-725, 2015.

18. Huang X, Wang X, Cheng C, Cai J, He S, Wang H, Liu F, Zhu C, Ding Z, Huang X, et al: Chaperonin containing TCP1, subunit 8 (CCT8) is upregulated in hepatocellular carcinoma and promotes HCC proliferation. APMIS 122: 1070-1079, 2014.

19. Sobin LH and Wittekind C; International Union Against Cancer (UICC): TNM Classification of Malignant Tumours. 5th edition. Sobin LH and Wittekind C (eds). Wiley-Liss, New York, NY, pp54-58, 1997.

20. Li M, Yang X, Zhang J, Shi H, Hang Q, Huang X, Liu G, Zhu J, $\mathrm{He} \mathrm{S}$ and Wang $\mathrm{H}$ : Effects of EHD2 interference on migration of esophageal squamous cell carcinoma. Med Oncol 30: 396, 2013.

21. Yu C, Chen K, Zheng H, Guo X, Jia W, Li M, Zeng M, Li J and Song L: Overexpression of astrocyte elevated gene-1 (AEG-1) is associated with esophageal squamous cell carcinoma (ESCC) progression and pathogenesis. Carcinogenesis 30: 894-901, 2009.

22. Yang X, Cheng L, Yao L, Ren H, Zhang S, Min X, Chen X, Zhang $\mathrm{J}$ and $\mathrm{Li} \mathrm{M}$ : Involvement of chromosome region maintenance 1 (CRM1) in the formation and progression of esophageal squamous cell carcinoma. Med Oncol 31: 155, 2014.

23. Valster A, Tran NL, Nakada M, Berens ME, Chan AY and Symons M: Cell migration and invasion assays. Methods 37: 208-215, 2005.

24. Wang D, He F, Zhang L, Zhang F, Wang Q, Qian X, Pan X, Meng J, Peng C, Shen A, et al: The role of p27(Kip1) phosphorylation at serine 10 in the migration of malignant glioma cells in vitro. Neoplasma 58: 65-73, 2011

25. Rodriguez LG, Wu X and Guan JL: Wound-healing assay. Methods Mol Biol 294: 23-29, 2005.

26. Llorca O, Martín-Benito J, Gómez-Puertas P, Ritco-Vonsovici M, Willison KR, Carrascosa JL and Valpuesta JM: Analysis of the interaction between the eukaryotic chaperonin CCT and its substrates actin and tubulin. J Struct Biol 135: 205-218, 2001.
27. Tamura T,Kuwahara A, Yamamori M,Nishiguchi K,Nakamura T, Okuno T, Miki I, Manabe Y and Sakaeda T: VEGF -634C/G genotype is predictive of long-term survival after treatment with a definitive 5-fluorouracil/cisplatin-based chemoradiotherapy in Japanese patients with esophageal squamous cell carcinoma. Int J Med Sci 9: 833-837, 2012.

28. Dekker C, Roe SM, McCormack EA, Beuron F, Pearl LH and Willison KR: The crystal structure of yeast CCT reveals intrinsic asymmetry of eukaryotic cytosolic chaperonins. EMBO J 30: 3078-3090, 2011.

29. Roobol A, Sahyoun ZP and Carden MJ: Selected subunits of the cytosolic chaperonin associate with microtubules assembled in vitro. J Biol Chem 274: 2408-2415, 1999.

30. Rademacher F, Kehren V, Stoldt VR and Ernst JF: A Candida albicans chaperonin subunit $(\mathrm{CaCct} 8 \mathrm{p})$ as a suppressor of morphogenesis and Ras phenotypes in C. albicans and Saccharomyces cerevisiae. Microbiology 144: 2951-2960, 1998.

31. Webb DJ,Zhang H and Horwitz AF: Cell migration: An overview. Methods Mol Biol 294: 3-11, 2005.

32. Stroud MJ, Kammerer RA and Ballestrem C: Characterization of G2L3 (GAS2-like 3), a new microtubule- and actin-binding protein related to spectraplakins. J Biol Chem 286: 24987-24995, 2011.

33. Llorca O, McCormack EA, Hynes G, Grantham J, Cordell J, Carrascosa JL, Willison KR, Fernandez JJ and Valpuesta JM: Eukaryotic type II chaperonin CCT interacts with actin through specific subunits. Nature 402: 693-696, 1999.

34. Llorca O, Martín-Benito J, Ritco-Vonsovici M, Grantham J, Hynes GM, Willison KR, Carrascosa JL and Valpuesta JM: Eukaryotic chaperonin CCT stabilizes actin and tubulin folding intermediates in open quasi-native conformations. EMBO J 19: 5971-5979, 2000

35. Tian G, Vainberg IE, Tap WD, Lewis SA and Cowan NJ: Quasinative chaperonin-bound intermediates in facilitated protein folding. J Biol Chem 270: 23910-23913, 1995.

36. Sternlicht H, Farr GW, Sternlicht ML, Driscoll JK, Willison K and Yaffe MB: The t-complex polypeptide 1 complex is a chaperonin for tubulin and actin in vivo. Proc Natl Acad Sci USA 90: 9422-9426, 1993.

37. Stoldt V, Rademacher F, Kehren V, Ernst JF, Pearce DA and Sherman F: Review: The Cct eukaryotic chaperonin subunits of Saccharomyces cerevisiae and other yeasts. Yeast 12: 523-529, 1996. 